\title{
Effect of UV curing conditions on polymerized tunable chiral nematic liquid crystals
}

\author{
Mohammad Mohammadimasoudi*a,b, Jeroen Beeckman ${ }^{\mathrm{a}, \mathrm{b}}$, Kristiaan Neyts ${ }^{\mathrm{a}, \mathrm{b}}$ \\ ${ }^{\mathrm{a}}$ ELIS Department, Ghent University, Gent, Belgium ${ }^{\mathrm{b}}$ Center for Nano- and Bio-photonics, Ghent \\ University, Ghent, Belgium
}

\begin{abstract}
Chiral nematic liquid crystals have attracted substantial interest. They spontaneously self-organize to form a helical structure with no complex fabrication procedure required and exhibit a reflection band for a certain wavelength interval. Since the photonic band gap can be tuned by applying external factors (heat, voltage, light, elasticity) chiral nematic liquid crystals are potentially interesting for large area optical filters and shutters, reflective displays and tunable lasers. In this work, a device which consists of a mixture of photo-polymerizable liquid crystal, non-reactive nematic liquid crystal and a chiral dopant is fabricated. By selecting the appropriate chiral dopant concentration, it is possible to make devices for different operation wavelengths. The influence of UV illumination on a partially polymerized chiral liquid crystal is investigated. A blue-wavelength shift of the photonic band gap is obtained as a function of power, duration time of UV illumination and the thickness of the cells. Interestingly the width and depth of the photonic band gap is unaffected by the change in UV curing conditions, which indicates that there is no degradation by the UV light.
\end{abstract}

Keywords: Liquid Crystal, UV curing, polymerization, tunable, optical filter

\section{INTRODUCTION}

Chiral nematic liquid crystals (CLC) have attracted considerable attention for their spontaneous arrangement into a helical periodic dielectric structure. The periodicity may be adapted such that a reflection band appears for visible wavelengths ${ }^{1}$. The periodicity of a CLC results in a 1D photonic band gap (PBG) with the band gap width of $\Delta \lambda=\Delta n P$ where $\Delta \mathrm{n}=$ ne-no is the LC birefringence and $\mathrm{P}$ is the pitch ${ }^{1,2}$. The band edges are defined by $P n_{o}<\lambda<P n_{e}$. CLCs are promising materials for electro-optic devices requiring low power, low cost and switchable diffracting elements such as lasers and displays ${ }^{3-8}$. In addition direct control and tuning of the PBG are of crucial importance for several emerging applications such as switchable optical devices, lab-on-a-chip devices as sensors ${ }^{9}$, modulators, reflectors and diffraction gratings. External stimuli can be used to tune the PBG such as heat ${ }^{10-13}$, light ${ }^{14-16}$, elasticity ${ }^{17}$ and voltage ${ }^{18-29}$. In previous work ${ }^{30}$ switchable devices with tuneable PBG with a fast response to electrical addressing and tuning over a broad range of wavelengths are demonstrated with a high reproducibility without deformation and degradation (Figure1). The shift is performed by applying an electric field over a layer consisting of a mixture of photopolymerizable LC and non-reactive nematic LC including a chiral dopant. In this work we investigate the effects of UV illumination on the same mixture. A blue wavelength shift of the PBG is obtained as a function of power, duration time of UV illumination and the thickness of the cells. There is no degradation and deformation by the change in UV curing conditions. It is well known that the use of photoresponsive chiral dopants results in a shift of the bandgap to longer wavelengths $s^{41}$. This is due to the fact that the chiral dopant loses its chiral twisting power after absorption of UV light. In this work we use a chiral dopant which does not react to UV light. Moreover, in this work the bandgap shifts to shorter wavelengths with increasing UV power, which is opposite to what would be expected from UV degradation of the chiral dopant.

*Mohammad.Mohammadimasoudi@elis.ugent.be; phone +32 9264 8952; fax +32 9264 3594; lcp.elis.ugent.be

Liquid Crystals XVIII, edited by lam Choon Khoo, Proc. of SPIE Vol. 9182, 91821A

(C) 2014 SPIE · CCC code: 0277-786X/14/\$18 · doi: 10.1117/12.2060965 




Figure 1. A photograph of a selective CLC reflector with a $1 \times 1.4 \mathrm{~cm} 2$ active region placed on a black background on which the word Mohammad is printed in white, a) without and b) with applied electric field.

\section{FABRICATION}

The CLC mixture is prepared by mixing $50 \mathrm{wt} \%$ non-reactive nematic LC (MDA-00-3536, Merck), $3.47 \mathrm{wt} \%$ right handed chiral dopant (BDH1305, Merck), $0.03 \mathrm{wt} \%$ photo initiator (Irgacure 819, BASF) and $46.5 \mathrm{wt} \%$ reactive LC in chloroform after stirring it for 20 minutes. The reactive LC is a blend of different reactive mono- and di-acrylate mesogens from Merck. It is possible to make devices for different operation wavelengths by selecting the appropriate chiral dopant concentration. A cell is made by two glass substrates with $30 \mathrm{~nm}$ thick conductive Indium-Tin-Oxide (ITO) electrodes. The substrates are coated with a nylon layer and rubbed anti-parallel in order to stabilize the CLC in the planar texture with the helical axis perpendicular to the glass substrates. The gap in the empty cell is determined by spacers balls mixed inside the glue. In this work different spacers of 4,8 or $10 \mu \mathrm{m}$ are used. The composite mixture is injected into the empty cell using the capillary effect in vacuum on a hot stage in the isotropic phase $\left(102^{\circ} \mathrm{C}\right)$ of the polymerizable LC mixture. The cell is cooled down such that the liquid crystal can orient itself into a helical structure as a uniform film without domains. Then the cell is exposed to UV light from a mercury lamp (with the main power at 365 $\mathrm{nm}$ and a blocking filter for short and long wavelengths) to polymerize the CLC mixture. A CLC polymer film is formed exhibiting selective reflection of right handed circularly polarized light. The CLC film is otherwise transparent without observable scattering. This indicates that droplets of nonreactive LC formed in the cross-linked network are much smaller than the wavelength of visible light (Figure 2). To control the behaviour of the CLC films, the UV power, curing time and the thickness of the cell are investigated. 


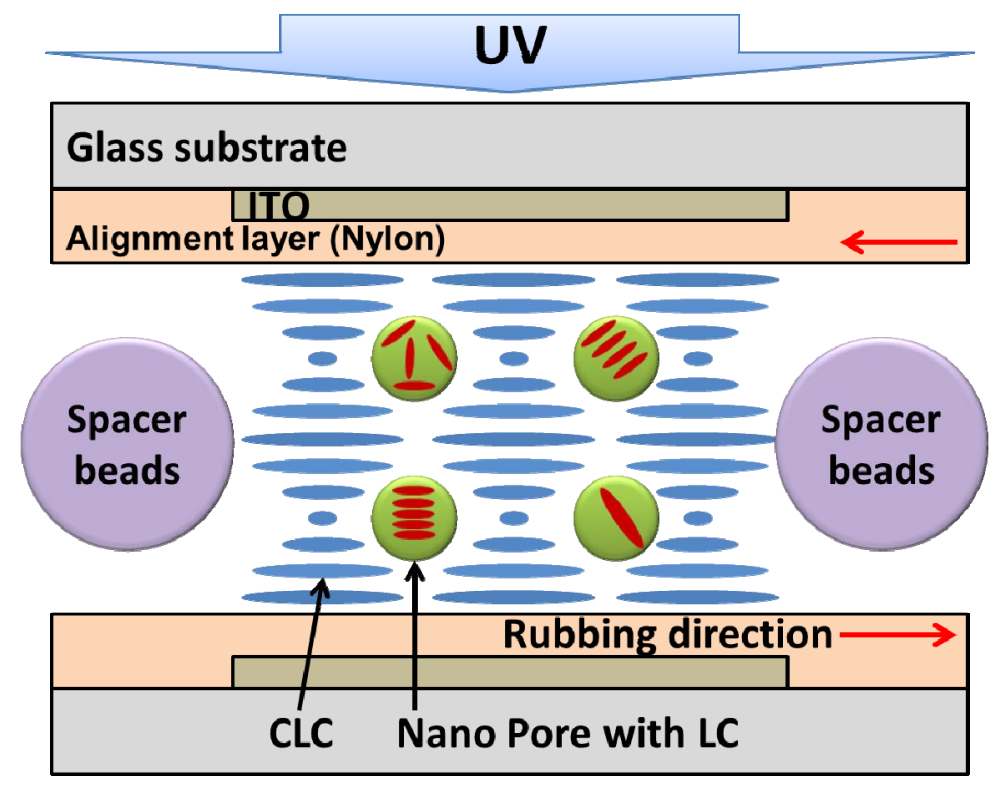

Figure 2. Scheme of the structure of a polymerized CLC network with dispersed nematic LC droplets.

\section{RESULTS AND DISCUSSION}

To investigate the influence of the UV power on the network structure and the photonic band gap, the mixture is infiltrated in cells with $8 \mu \mathrm{m}$ thickness. Then the cells are exposed to various powers of UV light $(1.75,3.5,7,10.5 \mathrm{~mW})$ for 1 minute to polymerize the CLC mixture. After fabrication, the transmission spectra of the cells are measured by a spectrometer (Perkin Elmer). In order to rule out the polarization dependencies of the spectrometer, the transmission measurements have been performed for right-handed circularly-polarized light produced with a linear polarizer and a zeroth-order quarter-wave plate at $850 \mathrm{~nm}$. The fabricated CLC films exhibit a broad PBG with a total bandwidth of approximately $100 \mathrm{~nm}$. The PBG of the devices shift to the blue by increasing the UV power as shown in figure 3(a). Various powers of UV light $(1.75,3.5,7,11,40 \mathrm{~mW})$ are used to illuminate the cells from both sides for a duration of 1 minute. The blue shift of the PBG of the devices is shown in figure 3(b) with increasing UV power. The strongest blue shift is $110 \mathrm{~nm}$ and the film features high stability and reflectivity without any noticeable degradation or disruption.
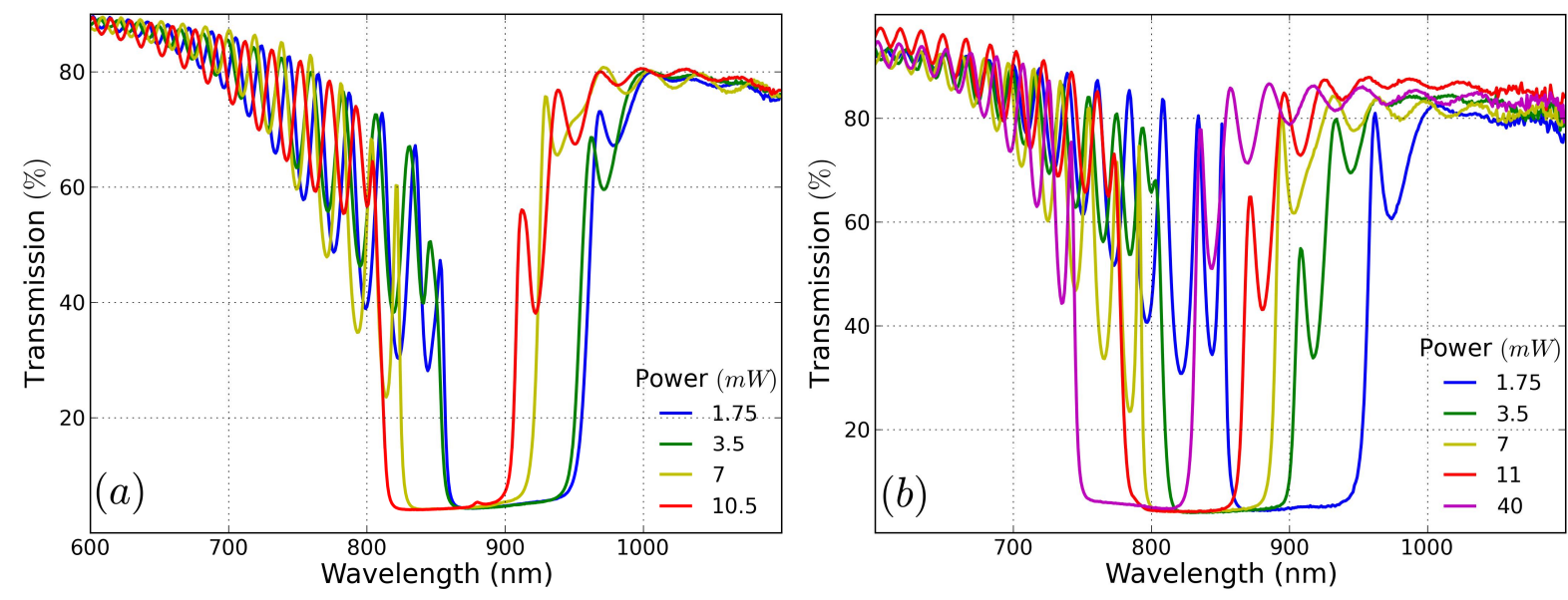

Figure 3. Transmission spectra (for right-handed circularly-polarized light) for devices exposed to various powers of UV light (365 nm) from a) one side and b) two sides 
To understand the reason for the shift, the refractive indices of the cells (without chiral dopant) are estimated for 2 powers of the UV light $(11$ and $40 \mathrm{~mW})$. The cells are illuminated from two sides for 1 minute. The blue shift corresponds to a decrease of the refractive indices as shown in figure 4.

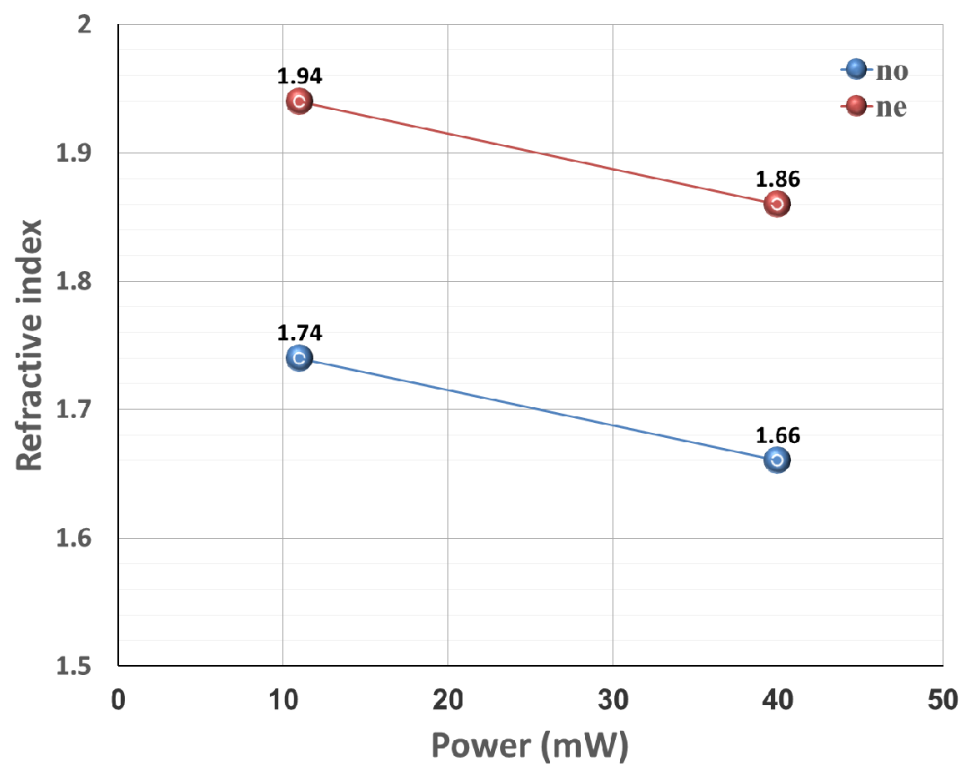

Figure 4. Ordinary and extra-ordinary refractive indices of devices exposed to two powers of UV light.

To investigate the effect of duration time of UV illumination on the network structure and photonic band gap, the cells with $8 \mu \mathrm{m}$ thickness are exposed to UV light with a power of $30 \mathrm{~mW}$ for 1 and 2 minutes. Figure 5 shows the blue shift of the PBG by applying UV light for these two durations. The shift is without degradation or disruption.



Figure 5. Transmission spectra (right handed circularly polarized light) for devices which are cured with $30 \mathrm{~mW}$ UV light (365 $\mathrm{nm})$ during 1 and 2 minutes.

Finally we have investigated the influence of the device thickness on the network structure and photonic band gap by injecting the mixture into the cells with 4, 8 and $10 \mu \mathrm{m}$ thicknesses. After being filled with the mixture, the cells are exposed to $30 \mathrm{~mW}$ UV light for 1 minute. The transmission spectra for these devices shown in figure 6 demonstrate that the PBG shifts to the red in thicker devices, or equivalently that the pitch increases in thicker cells. It is obvious that the 
transmission within the PBG decreases by increasing the thickness of the mixture ${ }^{32}$. The fact that the PBG shifts to the red with increasing thickness is most likely related to the fact that the average UV power over the thickness of the LC layer is smaller due to UV absorption.

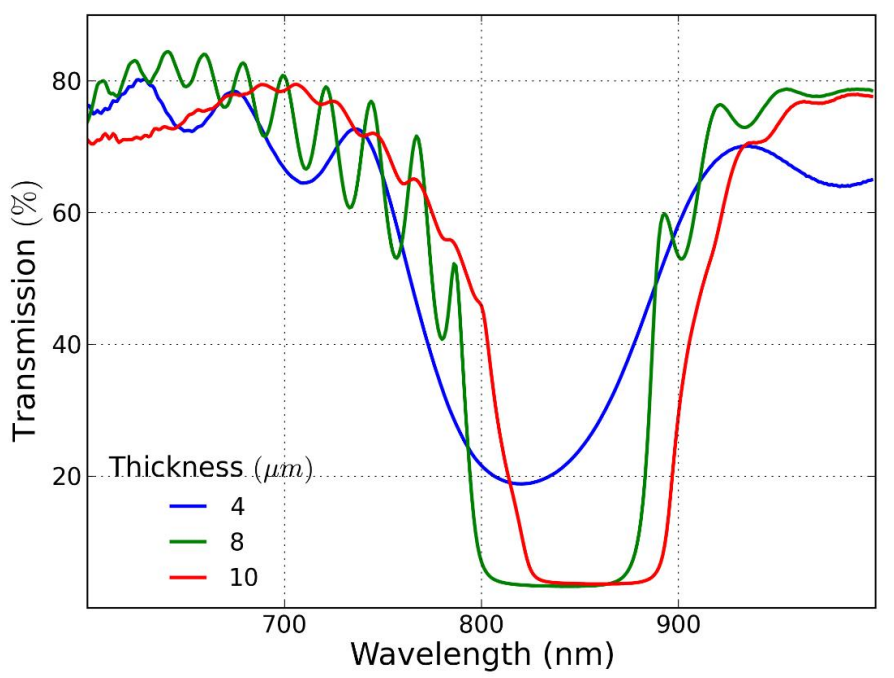

Figure 6. Transmission spectra (right-handed circularly-polarized light) for devices with various thicknesses which are illuminated by a $30-\mathrm{mW}$ UV light $(365 \mathrm{~nm})$ for 1 minute.

\section{CONCLUSION}

We have demonstrated a wide shift of the photonic band gap for a mixture of photo-polymerizable LC, nematic LC and a chiral dopant, by changing the power and time duration of the UV illumination and the thickness of the cell. The PBG can be shifted over $110 \mathrm{~nm}$ with relatively high stability and reflectivity, without any noticeable degradation or disruption. We can conclude that UV illumination plays an important role in controling the photonic band gap of the CLC mixture. The shift is due to a decrease in the refractive indices, but the physical mechanism for this decrease is not yet clear. Further experiments are required to find out the underlying mechanism.

\section{ACKNOWLEDGMENTS}

This research was supported by the Interuniversity Attraction Poles program of the Belgian Science Policy Office, under grant IAP P7-35.

\section{REFERENCES}

[1] P. G. De Gennes, [The Physics of Liquid Crystals] Clarendon Press, Oxford, (1974).

[2] D. K. Yang, J. L. West, L. C. Chien et al., "Control of reflectivity and bistability in displays using cholesteric liquid crystals," Journal of Applied Physics, 76(2), 1331-1333 (1994).

[3] V. A. Belyakov, "Low threshold DFB lasing at the edge and defect modes in chiral liquid crystals," Molecular Crystals and Liquid Crystals, 488, 279-308 (2008).

[4] A. Chanishvili, G. Chilaya, G. Petriashvili et al., "Lasing in dye-doped cholesteric liquid crystals: Two new tuning strategies," Advanced Materials, 16(9-10), 791-+ (2004). 
[5] H. Shirvani-Mahdavi, E. Mohajerani, and S. T. Wu, "Circularly polarized high-efficiency cholesteric liquid crystal lasers with a tunable nematic phase retarder," Optics Express, 18(5), 5021-5027 (2010).

[6] A. D. Ford, S. M. Morris, and H. J. Coles, "Phototonics and lasing in liquid crystals," Materials Today, 9(7-8), 36-42 (2006).

[7] L. Penninck, J. Beeckman, P. De Visschere et al., "Numerical simulation of stimulated emission and lasing in dye doped cholesteric liquid crystal films," Journal of Applied Physics, 113(6), (2013).

[8] Y. Inoue, H. Yoshida, K. Inoue et al., "Improved Lasing Threshold of Cholesteric Liquid Crystal Lasers with In-Plane Helix Alignment," Applied Physics Express, 3(10), (2010).

[9] S. Kado, Y. Takeshima, Y. Nakahara et al., "Potassium-ion-selective sensing based on selective reflection of cholesteric liquid crystal membranes," Journal of Inclusion Phenomena and Macrocyclic Chemistry, 72(1-2), 227-232 (2012).

[10] Y. H. Huang, Y. Zhou, C. Doyle et al., "Tuning the photonic band gap in cholesteric liquid crystals by temperature-dependent dopant solubility," Optics Express, 14(3), 1236-1242 (2006).

[11] K. Funamoto, M. Ozaki, and K. Yoshino, "Discontinuous shift of lasing wavelength with temperature in cholesteric liquid crystal," Japanese Journal of Applied Physics Part 2-Letters, 42(12B), L1523-L1525 (2003).

[12] L. V. Natarajan, J. M. Wofford, V. P. Tondiglia et al., "Electro-thermal tuning in a negative dielectric cholesteric liquid crystal material," Journal of Applied Physics, 103(9), 7 (2008).

[13] S. Furumi, and N. Tamaoki, "Glass-Forming Cholesteric Liquid Crystal Oligomers for New Tunable SolidState Laser," Advanced Materials, 22(8), 886-+ (2010).

[14] S. Kurihara, Y. Hatae, T. Yoshioka et al., "Photo-tuning of lasing from a dye-doped cholesteric liquid crystals by photoisomerization of a sugar derivative having plural azobenzene groups," Applied Physics Letters, 88(10), (2006).

[15] G. S. Chilaya, "Light-controlled change in the helical pitch and broadband tunable cholesteric liquid-crystal lasers," Crystallography Reports, 51, S108-S118 (2006).

[16] T. J. White, R. L. Bricker, L. V. Natarajan et al., "Electromechanical and light tunable cholesteric liquid crystals," Optics Communications, 283(18), 3434-3436 (2010).

[17] H. Finkelmann, S. T. Kim, A. Munoz et al., "Tunable mirrorless lasing in cholesteric liquid crystalline elastomers," Advanced Materials, 13(14), 1069-+ (2001).

[18] H. P. Yu, B. Y. Tang, J. H. Li et al., "Electrically tunable lasers made from electro-optically active photonics band gap materials," Optics Express, 13(18), 7243-7249 (2005).

[19] Y. Inoue, Y. Matsuhisa, H. Yoshida et al., "Electric Field Dependence of Lasing Wavelength in Cholesteric Liquid Crystal with an In-Plane Helix Alignment," Molecular Crystals and Liquid Crystals, 516, 182-189 (2010).

[20] H. Yoshida, Y. Inoue, T. Isomura et al., "Position sensitive, continuous wavelength tunable laser based on photopolymerizable cholesteric liquid crystals with an in-plane helix alignment," Applied Physics Letters, 94(9), (2009).

[21] S. S. Choi, S. M. Morris, W. T. S. Huck et al., "Electrically Tuneable Liquid Crystal Photonic Bandgaps," Advanced Materials, 21(38-39), 3915-+ (2009).

[22] S. S. Choi, S. M. Morris, W. T. S. Huck et al., "Simultaneous red-green-blue reflection and wavelength tuning from an achiral liquid crystal and a polymer template," Advanced Materials, 22(1), 53-+ (2010).

[23] B. Park, M. Kim, S. W. Kim et al., "Electrically Controllable Omnidirectional Laser Emission from a HelicalPolymer Network Composite Film," Advanced Materials, 21(7), 771-+ (2009).

[24] Y. Inoue, H. Yoshida, K. Inoue et al., "Tunable Lasing from a Cholesteric Liquid Crystal Film Embedded with a Liquid Crystal Nanopore Network," Advanced Materials, 23(46), 5498-+ (2011).

[25] B. W. Liu, Z. G. Zheng, X. C. Chen et al., "Low-voltage-modulated laser based on dye-doped polymer stabilized cholesteric liquid crystal," Optical Materials Express, 3(4), 519-526 (2013).

[26] J. Schmidtke, G. Junnemann, S. Keuker-Baumann et al., "Electrical fine tuning of liquid crystal lasers," Applied Physics Letters, 101(5), (2012).

[27] C. A. Bailey, V. P. Tondiglia, L. V. Natarajan et al., "Electromechanical tuning of cholesteric liquid crystals," Journal of Applied Physics, 107(1), 013105 (8 pp.)-013105 (8 pp.) (2010).

[28] S. S. Choi, S. M. Morris, W. T. S. Huck et al., "The switching properties of chiral nematic liquid crystals using electrically commanded surfaces," Soft Matter, 5(2), 354-362 (2009).

[29] S. Furumi, S. Yokoyama, A. Otomo et al., "Control of photonic bandgaps in chiral liquid crystals for distributed feedback effect,” Thin Solid Films, 499(1-2), 322-328 (2006). 
[30] M. Mohammadimasoudi, J. Beeckman, J. Shin et al., "Widely tunable chiral nematic liquid crystal optical filter with microsecond switching time," Optics Express, 22(16), 19098-19107 (2014).

[31] A. Bobrovsky, and V. Shibaev, "Novel type of combined photopatternable and electro-switchable polymerstabilized cholesteric materials," Journal of Materials Chemistry, 19(3), 366-372 (2009).

[32] T. Scharf, [Polarized light in liquid crystals and polymers] John Wiley \& Sons, Inc, United States of America, 365 (2007). 